\title{
Ptychographic X-Ray Imaging of Colloidal Crystals
}

\author{
Sergey Lazarev, Ilya Besedin, Alexey V. Zozulya, Janne-Mieke Meijer, \\ Dmitry Dzhigaev, Oleg Yu. Gorobtsov, Ruslan P. Kurta, Max Rose, Anatoly G. Shabalin, \\ Elena A. Sulyanova, Ivan A. Zaluzhnyy, Alexey P. Menushenkov, Michael Sprung, \\ Andrei V. Petukhov, and Ivan A. Vartanyants*
}

Ptychographic coherent X-ray imaging is applied to obtain a projection of the electron density of colloidal crystals, which are promising nanoscale materials for optoelectronic applications and important model systems. Using the incident X-ray wavefield reconstructed by mixed states approach, a high resolution and high contrast image of the colloidal crystal structure is obtained by ptychography. The reconstructed colloidal crystal reveals domain structure with an average domain size of about $2 \mu \mathrm{m}$. Comparison of the domains formed by the basic close-packed structures, allows us to conclude on the absence of pure hexagonal close-packed domains and confirms the presence of random hexagonal close-packed layers with predominantly face-centered cubic structure within the analyzed part of the colloidal crystal film. The ptychography reconstruction shows that the final structure is complicated and may contain partial dislocations leading to a variation of the stacking sequence in the lateral direction. As such in this work, X-ray ptychography is extended to high resolution imaging of crystalline samples.

dipolar interactions. ${ }^{[1-15]}$ The flexibility of colloidal crystals and their response to external stimuli ${ }^{[16-18]}$ along with their unique optical properties such as the strongly pronounced structural color and photonic band gap makes them attractive for many applications. ${ }^{[19-25]}$ Moreover, defects, which determine the mechanical properties of many engineering materials such as metals, ${ }^{[26]}$ can be studied with "atomic" resolution using colloidal crystals. ${ }^{[27-29]}$

The high penetration depth of X-rays makes them an ideal tool to access important statistically averaged spatial information about the colloidal crystal structure and disorder over macroscopic sample volume..$^{[7,30,31]}$ Further access to local structure of colloidal crystals can be gained by using microscopy with Fresnel optics and soft X-rays, ${ }^{[32,33]}$ but unfortunately the use

\section{Introduction}

Colloidal crystals are being actively employed as an important model system to study melting, freezing, and solid-solid phase transitions as a function of osmotic pressure and anisotropy of interparticle interaction due to particle shape, patchiness, or of soft X-rays significantly limits the penetration depth. Alternatively, compound refractive lenses (CRLs) can be used as an objective lens for hard X-rays to study thicker samples. ${ }^{[34-36]}$ This technique provides full field of view, however, resolution is limited by the resolving power of the optical elements and contrast is limited by using hard X-rays. ${ }^{[37]}$
Dr. S. Lazarev, I. Besedin, ${ }^{[+]}$Dr. A. V. Zozulya, ${ }^{[+]}$Dr. D. Dzhigaev,

Dr. O. Yu. Gorobtsov, Dr. R. P. Kurta, ${ }^{[+]}$M. Rose, Dr. A. G. Shabalin,,$^{[+++]}$

Dr. I. A. Zaluzhnyy, Dr. M. Sprung, Prof. I. A. Vartanyants

Deutsches Elektronen-Synchrotron DESY

Notkestraße 85, D-22607 Hamburg, Germany

E-mail: ivan.vartaniants@desy.de

S. Lazarev

National Research Tomsk Polytechnic University (TPU)

pr. Lenina 30, 634050 Tomsk, Russia

The ORCID identification number(s) for the author(s) of this article can be found under https://doi.org/10.1002/smll.201702575.

${ }^{[+]}$Present address: National University for Science and Technology (MISiS), Leninskiy pr. 4, Moscow 119049, Russia

${ }^{[++]}$Present address: European XFEL GmbH, Holzkoppel 4, D-22869

Schenefeld, Germany

${ }^{[+++] P r e s e n t ~ a d d r e s s: ~ S o f t ~ M a t t e r ~ P h y s i c s, ~ U n i v e r s i a ̈ t ~ K o n s t a n z, ~ D-78457 ~}$ Konstanz, Germany

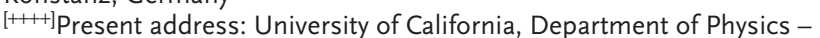

San Diego, 92093 La Jolla, USA

DOI: 10.1002/smll.201702575
I. Besedin, Dr. I. A. Zaluzhnyy, Prof. A. P. Menushenkov, Prof. I. A. Vartanyants National Research Nuclear University

MEPhl (Moscow Engineering Physics Institute)

Kashirskoe shosse 31, 115409 Moscow, Russia

Dr. J.-M. Meijer, ${ }^{[++]}$Dr. A. V. Petukhov

Van't Hoff Laboratory for Physical and Colloid Chemistry

Debye Institute for Nanomaterial Science

Utrecht University

Padualaan 8, $3584 \mathrm{CH}$ Utrecht, The Netherlands

Dr. E. A. Sulyanova

Shubnikov Institute of Crystallography RAS

Leninskii pr. 59, 119333 Moscow, Russia

Dr. A. V. Petukhov

Laboratory of Physical Chemistry

Department of Chemical Engineering and Chemistry

Eindhoven University of Technology

P.O. Box 513, 5600 MB Eindhoven, Netherlands 
The development of third-generation synchrotron sources provides new perspectives for high-resolution X-ray imaging using coherent X-ray diffraction imaging (CXDI) technique (see for review ${ }^{[38]}$. CXDI reveals the electron density distribution in nano- and mesoscale objects with the resolution limited only by the scattering angle and tolerable signal to noise ratio. In contrast to X-ray crystallography, CXDI does not require the sample to be crystalline. It allows determination of the strain field distribution formed by defects in a nonperfect crystalline material. CXDI was successfully applied for high resolution imaging of defects in colloidal crystal films and colloidal crystal grains. ${ }^{[39-41]}$ At the same time, conventional CXDI relies on a sample smaller than the incident beam size and a high degree of spatial coherence of X-rays. In order to obtain a large field of view for such mesoscale materials as colloidal crystal films, another high resolution coherent scattering method can be used, namely ptychography.

X-ray ptychography is a variation of an X-ray scanning imaging technique in which the sample is scanned across the beam with overlapping footprints of the probe illumination. ${ }^{[42,43]}$ Instead of a sample being smaller than the beam size, it exploits the small size of a focused coherent X-ray beam illuminating the sample. At each scanning position a diffraction pattern in the far-field is recorded. Due to the spatial overlap of the illuminated parts of the specimen, each position on the sample contributes to a multitude of different diffraction patterns that provides faster and more stable convergence of the reconstruction procedure. As an advantage over the CXDI, ptychography also retrieves the complex valued illumination function. ${ }^{[4]}$

Originally, ptychography was proposed as an atomic resolution technique for electron scanning microscopy. ${ }^{[45-47]}$ Recently, substantial improvement in resolution has been achieved in electron ptychographic imaging of crystalline samples (see, for example, ref. [48]). At the same time, most of X-ray ptychography applications were focused on noncrystalline samples. ${ }^{[49]}$ Here we extend X-ray ptychography work by applying it to high resolution imaging of a crystalline colloidal film.

\section{Results}

The scheme of the ptychographic experiment is presented in Figure 1. The thin colloidal crystal film that consisted of polystyrene (PS) colloidal particles of $418 \mathrm{~nm}$ in diameter (see the Experimental Section for details) was positioned perpendicular to the incoming X-ray beam. The colloidal crystal sample was scanned over an area of 10 by $10 \mu \mathrm{m}^{2}$. The detector was positioned in the far-field downstream from the sample. Scattering of X-rays on a hexagonal colloidal crystal leads to formation of diffraction patterns with the six fold symmetry, as it is shown in Figure 1.

A typical diffraction pattern from the ptychographic scan of the colloidal crystal is shown in Figure 2a. A large number of Bragg peaks up to the third order are well resolved in this pattern. The corresponding [10.0] and [11.0] crystallographic directions are shown in this figure as white arrows. The most intense Bragg peaks in our measurements were the 11.0 reflections. The 10.0 reflections are very close to the direct beam and were slightly affected by the diffuse scattering from the semitransparent beamstop. The broadening of the Bragg peaks may be attributed to coherent illumination of the sample as well as to defect structure of the colloidal crystal (see, for example, ref. [50]).

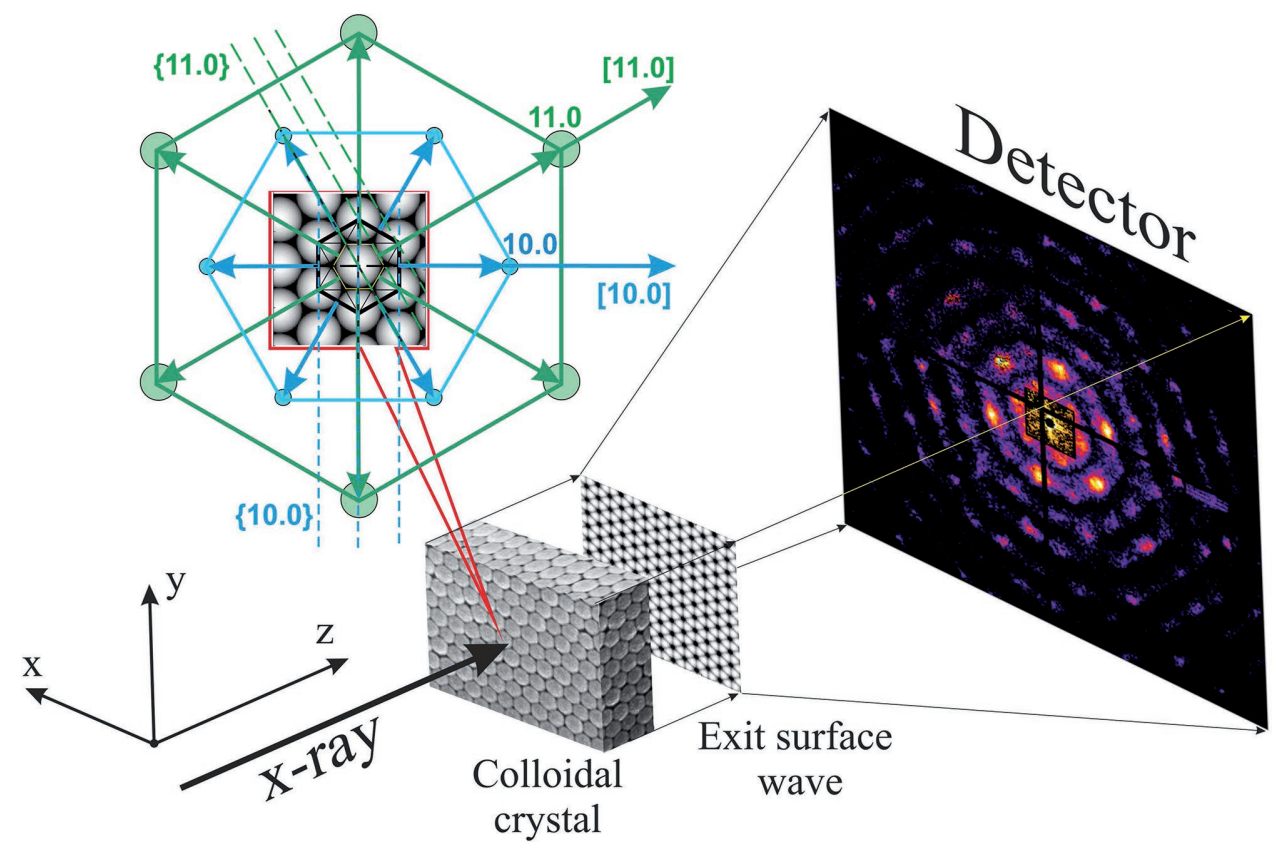

Figure 1. Scheme of the ptychographic experiment on a colloidal crystal sample. The surface of the colloidal sample is perpendicular to the incoming X-ray beam. The diffraction of X-rays on the crystalline structure of the film leads to formation of Bragg peaks at the detector. The Cartesian coordinate system with the $z$-axis taken along the optical axis of the X-ray beam and $x, y$ axes taken in transverse direction is also shown. (Inset) Corresponding diffraction planes (shown by dashed lines), crystallographic directions and Bragg peaks are colored in green for [11.0] and in blue for [10.0] crystallographic directions of the colloidal crystal. 


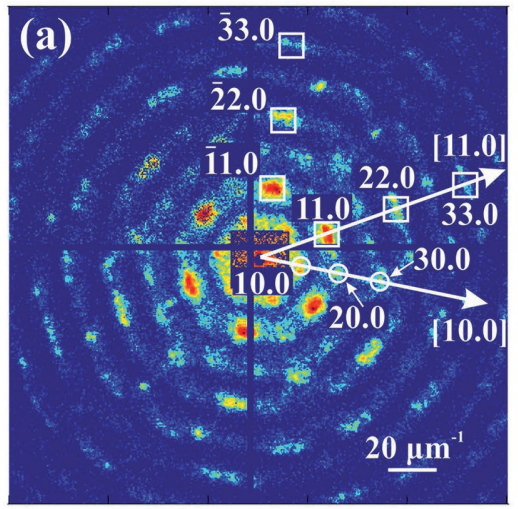

(c)

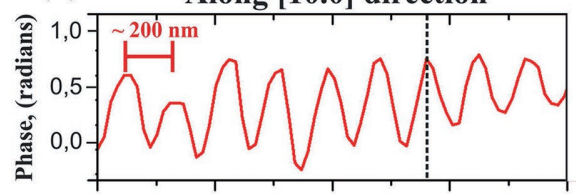

Along [11.0] direction

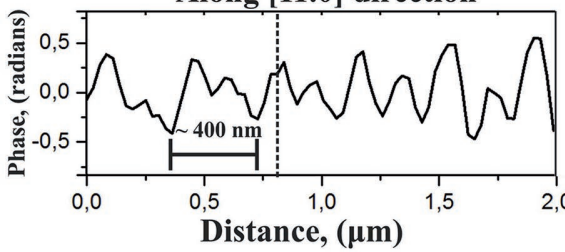

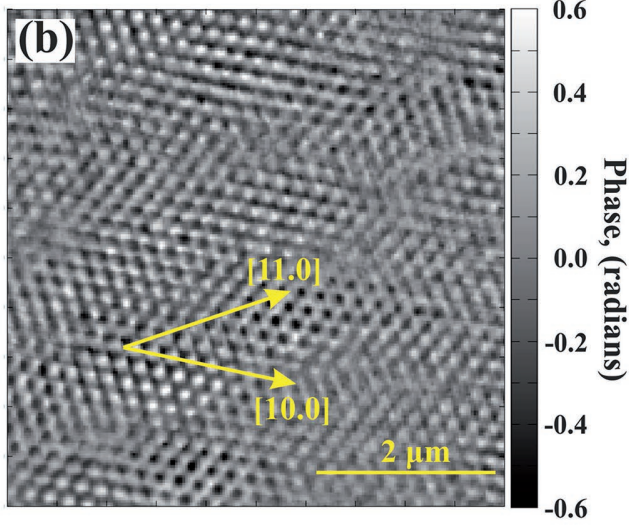

(d)

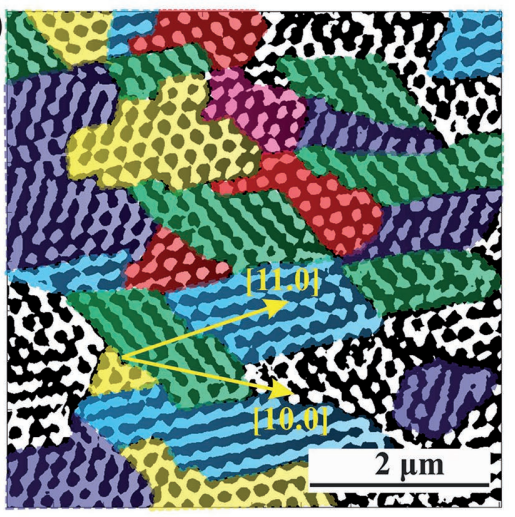

Figure 2. a) Typical diffraction pattern from the ptychographic data set. White arrows indicate [10.0] and [11.0] crystallographic directions. b) Reconstructed phase of the colloidal crystal object function $O(x, y)$. Yellow arrows indicate [10.0] and [11.0] crystallographic directions, along which profiles of the phase were determined and are shown in (c). For each direction, an average period of the phase profile is indicated, and is about $200 \mathrm{~nm}$ and $400 \mathrm{~nm}$ for the [10.0] and [11.0] crystallographic directions, respectively. d) Domain structure of the reconstructed phase map of the crystal revealed by the filled contour plot. Yellow, red and purple areas are attributed to the domains with the dominant fcc and the green, blue and pink domains are identified as rhcp structures. Not classified areas are not colored. Yellow arrows in (d) correspond to the same crystallographic directions as in (b). The dashed vertical lines in (c) indicate domain borders in (d).

Ptychographic reconstruction of the colloidal sample was performed in two steps. First, the incident wave field was reconstructed from the ptychographic measurements using the Siemens star test gold pattern. This reconstruction was performed by employing the mixed state approach, ${ }^{[51]}$ where the initial probe function was represented as a superposition of five Hermite-Gaussian modes (see the Supporting Information for details). Using this probe function, a significant improvement of the final colloidal crystal reconstruction was achieved.

In the next step, the complex-valued object function $O(x, y)$ corresponding to the projected electron density $R(x, y)$ of the colloidal crystal (see section Theory of Ptychographic Experiment) was ptychographically reconstructed. In the reconstruction, 5000 iterations of the alternating projections algorithm were used ${ }^{[52]}$ that provided sufficiently low error metric. The phase of the object function is presented in Figure $2 \mathrm{~b}$. In the reconstructed image, the pixel size was $29.3 \mathrm{~nm}$, which was fine enough to resolve details of the phase variation.

The hexagonal structure expected from a close-packing of spheres can be well observed in the reconstructed phase of the complex object function (see Figure $2 \mathrm{~b}$ ). We can also see that the orientation of the close-packed structure is well preserved across the object over a large distance, while at the same time the contrast varies along the surface. Such varying contrast may be attributed to the presence of different domains and defects in the colloidal crystal structure. As soon as 2D ptychography reconstruction gives a projection image, the full characterization of defects is not feasible in this case (compare to 3D reconstruction of the colloidal crystal grain in ref. [41], where all defects were identified without ambiguity).

\section{Discussion}

We will start our discussion with an overview of the closepacked structures. A projection of a single hexagonal closepacked layer with the colloidal particles of diameter $d$ is shown in Figure 3a. Due to hexagonal symmetry, there are two fundamental crystallographic directions: [10.0] along $x$ axis and [11.0] through the centers of the nearest neighbor colloidal particles in $30^{\circ}$ to the [10.0] direction. We adopted indexing from the hexagonal close-packed (hcp) structure classification with the point symbolizing the fourth index equal to the negative sum of the first two indexes (11.0 equals to $11 \overline{2} 0)$. The profiles of the projected electron density $R(x, y)$ along these two crystallographic directions are shown in Figure $3 \mathrm{~d}$. These profiles 

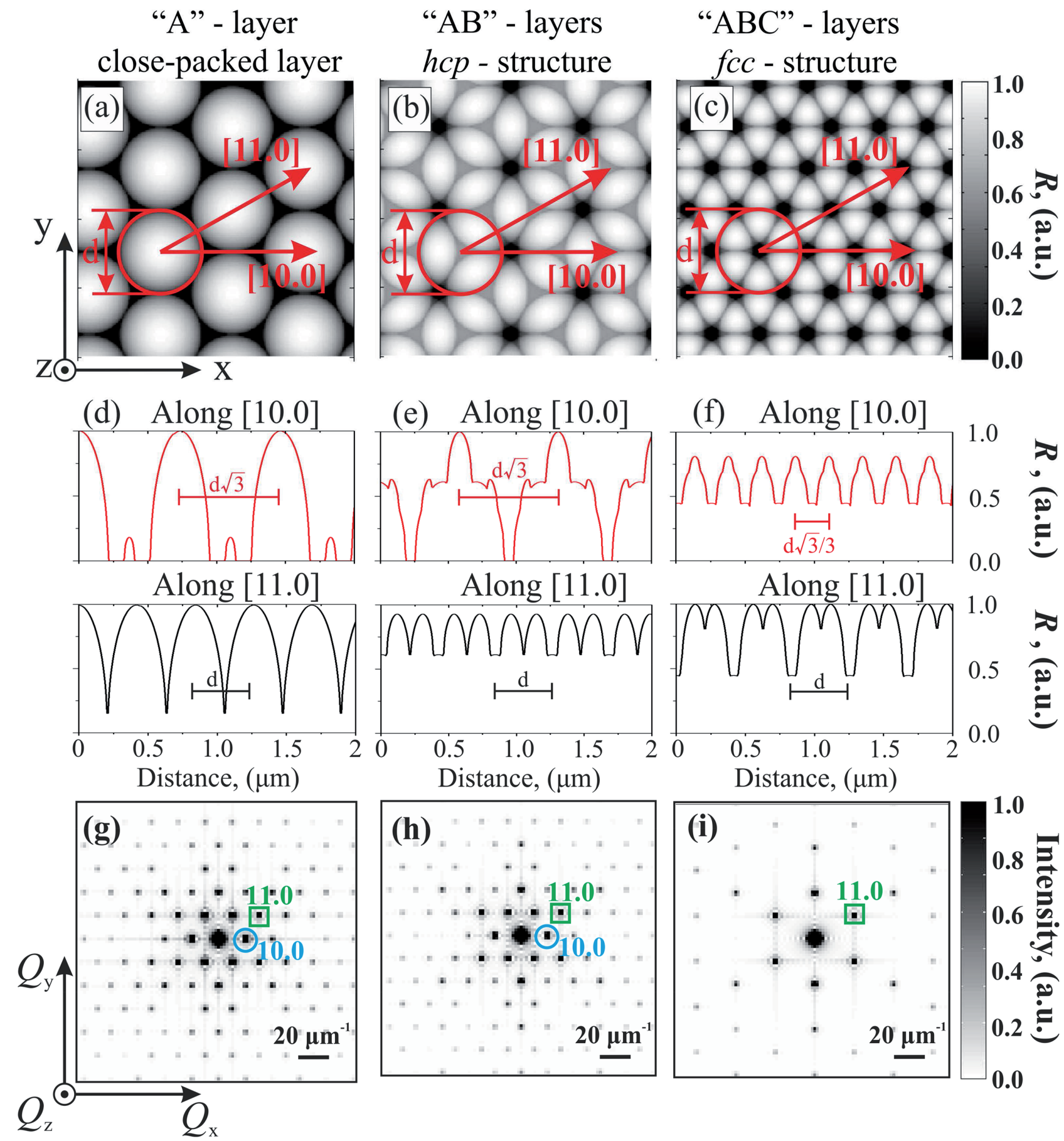

Figure 3. Projections of different close-packed structures. a) Single close-packed layer ("A"-layer), b) hexagonal close-packed hcp structure, or "AB", c) face centered cubic fcc structure, or "ABC," $d-f$ ) profiles of the optical density along [10.0] and [1 1.0] crystallographic directions of corresponding close-packed structures. Periods of corresponding structures are shown and are equal to $d \sqrt{3}$ and $d$ for [10.0] and [11.0] directions, respectively. For [10.0] direction of the fcc "ABC" structure this period is equal to $d \sqrt{3} / 3$. g-i) Diffraction patterns of the close-packed structures corresponding to their projections shown in $(\mathrm{a}-\mathrm{c})$.

are periodic functions with the periods equal to $d \sqrt{3}$ and $d$ for [10.0] and [11.0] directions, respectively.

A system of diffraction peaks originating from a single layer of such a close-packed structure is shown in Figure $3 \mathrm{~g}$ and in the inset of Figure 1. Bragg reflections are formed in the directions of [10.0] and [11.0] perpendicular to the planes $\{10.0\}$ and $\{11.0\}$. The distance between neighboring planes of these sets are ${ }^{[53]} d_{10.0}=d \sqrt{3} / 2$ and $d_{11.0}=d / 2$. Corresponding reciprocal lattice vectors are given by $Q_{10.0}=2 \pi / d_{10.0}$ and $Q_{11.0}=$ $2 \pi / d_{11.0}$, which means that the set of 10.0 Bragg reflections is 
closest to the origin of reciprocal space and the set of 11.0 Bragg peaks is rotated by $30^{\circ}$ with respect to 10.0 peaks and appear $\sqrt{3}$ times further away from the origin of reciprocal space (see Figure $3 \mathrm{~g}$ and inset in Figure 1).

It is well known that further stacking of layers will result in two elementary close-packed structures: "AB" that is hexagonal close-packed (hcp structure and "ABC" that is face-centered cubic (fcc) structure (see for projection of corresponding structures Figure $3 \mathrm{~b}, \mathrm{c}){ }^{[54]}$ The profiles of the electron density projection of these structures along the [10.0] and [11.0] directions are presented in Figure 3e,f for hcp and fcc structures, respectively. Both hcp and fcc structures have the period equal to $d$ along [11.0] direction. Along [10.0] direction hcp structure has a period equal to $d \sqrt{3}$ and fcc structure has a period of $d \sqrt{3} / 3$. It is clear from these figures that already these basic structures have complicated projections and profiles of $R(x, y)$ with local minima in the center of colloidal particles of the layer "A".

Diffraction patterns corresponding to hcp and fcc structures are shown in Figure 3h,i. We can see that in general 11.0 reflections are stronger than 10.0. In the case of an fcc structure, 10.0 reflections are forbidden for infinitely large and defect free colloidal crystal and only 11.0 Bragg peaks are present.

The free energy difference between fcc and hcp structures of colloidal crystals of hard spheres is very small. ${ }^{[5]}$ Therefore, during the self-organized growth of colloidal crystal film, a mixture of these two lattices is often formed in equilibrium structures. This mixture of packing layers is called a random hexagonal close-packed (rhcp) structure. ${ }^{[53]}$

Typical colloidal crystal systems consist of many close-packed layers. By discussing two "AB" layers or three "ABC" layers we assume that, if they are continued to form a thick colloidal crystal film, in projection they will give the same structure as shown above. This is different for rhcp structure where projection may be formed by a dominant hcp or fcc structure (see the Supporting Information).

Description of these basic close-packed structures allowed us to quantify results of the ptychographic experiment. The phase of the reconstructed complex-valued object function shown in Figure $2 \mathrm{~b}$ is proportional to projection of the electron density $R(x, y)$ along the beam propagation (see Section Theory of Ptychographic Experiment). The profiles of the phase for [10.0] and [11.0] crystallographic directions, shown as yellow arrows in Figure 2b, are presented in Figure 2c.

The high contrast periodic pattern was observed in both crystallographic directions. The average period of the $R(x, y)$ function presented in Figure $2 \mathrm{c}$ is about $200 \pm 50 \mathrm{~nm}$ and $400 \pm 50 \mathrm{~nm}$ for the [10.0] and [11.0] crystallographic directions, respectively. Since the colloidal spheres in our sample have a diameter of $418 \mathrm{~nm}$, the expected period along [11.0] crystallographic direction for the rhcp structure consisting of fcc and hcp layers is $418 \mathrm{~nm}$, which is in good agreement with our observations (400 $\mathrm{nm}$ along [11.0] direction). The period in the [10.0] direction for different domains is $200 \mathrm{~nm}$, which excludes pure hcp structure (see Figure 3), exhibiting the period of $724 \mathrm{~nm}$ in this direction.

In addition, we observed a slight variation of this period along the surface of the colloidal crystal due to imperfections of the sample. Our analysis of the rhcp structure (see the Supporting Information for details) shows that for a dominant contribution of fcc stacking to the rhcp structure the period is close to the one observed in the sample (it is $241 \mathrm{~nm}$ for pure fcc structure). As such, in our experiment we observe an rhcp structure with possible preference for fcc sequences (see the Supporting Information for details), as observed before in refs. [50,56].

The 2D phase of the reconstructed object function $O(x, y)$ is shown in Figure 2d as a filled contour plot. Visual inspection of this contour plot revealed different domains in the colloidal crystal electron density projection $R(x, y)$. The specifically identified and classified domains are indicated with different colors in Figure $2 \mathrm{~d}$. The parts that were difficult to classify were left uncolored. The yellow, red and purple areas indicate domains with dominant fcc ("ABC") structure based on the similarity of the electron density projection $R(x, y)$ of the fcc structure (see Figure $3 c$ and the Supporting Information). The green, blue and pink domains are identified as rhcp structure. These regions are separated by partial dislocations leading to a variation of the lateral position while keeping the overall layer orientation the same. ${ }^{[28,57]}$ As discussed above, the absence of equal periodicity along the whole reconstructed phase map means that domains with a pure hcp structure are absent in the explored region of the colloidal crystal film.

The presence of many differently stacked domains in a close proximity can be attributed to stacking faults and partial dislocations, and has been discussed in detail. ${ }^{[28,58]}$ Interestingly, such fine domain structure was not observed before ${ }^{[53]}$ neither by the optical nor by scanning electron microscopy (SEM). Hard X-ray ptychography, being highly penetrating technique, provides different contrast mechanism ${ }^{[37]}$ in comparison to SEM and reveals complementary features in colloidal crystal samples. The average domain size as seen in Figure $2 d$ is of the order of $2 \mu \mathrm{m}$, which is in good agreement with the domain sizes obtained for similar colloidal crystal films using reciprocal space analysis. ${ }^{[50]}$ At the same time, the periodicity in the phase profiles changes only slightly as they cross domain boundaries in Figure 2c, which are shown as dashed vertical lines. This feature may indicate that the observed defects are partial dislocations. ${ }^{[28,58]}$ Such dislocations may be described as transition between the stacking sequences of 3 layers with a shift from local fcc via hcp to fcc with different stacking direction: (ABC via ABA to $\mathrm{CBA}$ ). Clearly, the colloidal crystal studied here contains many of these transitions. Why these transitions occur is presently unclear. As it was found in a previous study, ${ }^{[59]}$ in a convective assembly method a growth front is formed which should favor dominant fcc structure. Clearly, the ptychography reconstruction shows that the final structure is more complicated and may contain partial dislocations leading to a variation of the stacking sequence in the lateral direction and, hence, that the formation process is not completely understood yet. Therefore, further studies are obviously needed to reveal more details including the structure of these partial dislocations.

\section{Conclusions}

Ptychographic coherent imaging was applied to determine the projected electron density of a polystyrene colloidal crystal film. The ptychographic experiment was performed in two steps. In the first step the incoming complex wave field was reconstructed employing the mixed state approach and using a 
Siemens star test pattern. In the next step, colloidal crystal film structure was reconstructed, using the determined previously incoming wave field, with high resolution and contrast.

The experimentally determined structure of the colloidal crystal revealed domains with an average size of about $2 \mu \mathrm{m}$. Comparison of the inner structure of domains with the basic closed-packed structures, composed of spheres, allowed us to conclude on the absence of pure hcp domains and confirmed presence of rhcp domains with predominantly fcc structure within the investigated part of the colloidal crystal film. We also observed a large amount of domain boundaries which are possibly organized by partial dislocations.

The present experiment outlines an approach for quantitative high resolution and high contrast colloidal crystal film characterization of practically unlimited lateral size by ptychographic coherent X-ray diffractive imaging. This technique provides a unique possibility to image evolution of the defect structure in colloidal crystal films. That may be very attractive, for example, for in situ studies of solid-solid phase transitions. By our work we extend X-ray ptychography that is a high resolution coherent imaging technique to studies of crystalline structures on a mesoscale. Moreover, it demonstrates possibility of large scale and atomic resolution ptychographic imaging of a crystalline structure using high energy coherent X-rays. ${ }^{[60]}$

\section{Theory and Experimental Section}

Theory of Ptychographic Experiment: Propagation of X-rays through the colloidal crystal can be described by the paraxial wave equation, which in Cartesian coordinates can be presented as ${ }^{[36,61]}$

$-2 i k \frac{\partial E(\boldsymbol{r})}{\partial z}=\Delta_{\perp} E(\boldsymbol{r})+k^{2} \chi(\boldsymbol{r}) E(\boldsymbol{r})$

where $E(\boldsymbol{r})$ is the complex-valued wave amplitude, $k=2 \pi / \lambda$ is the wavenumber, $\lambda$ is the wavelength of $\mathrm{X}$-rays, $\Delta_{\perp}$ is the transverse part of the Laplace operator and $\chi(\boldsymbol{r})$ is the complex-valued susceptibility of the sample. Here the following coordinate system was adopted with the $z$ axis along the optical axis of a narrowly collimated coherent $X$-ray beam and $\boldsymbol{r}=\{x, y\}$ is a 2D vector in transverse direction along the surface of the colloidal crystal film, as shown in Figure 1.

Ptychography is based on the assumption that the interaction of the incident X-ray beam with the sample can be described in the projection approximation. ${ }^{[62]}$ In the frame of this approximation the exit surface wave (ESW), that represents the wave directly behind the sample (see Figure 1), may be described by the following relation

$E_{\mathrm{ESW}}\left(\boldsymbol{r}, \boldsymbol{r}^{\prime}\right)=P\left(\boldsymbol{r}-\boldsymbol{r}^{\prime}\right) O\left(\boldsymbol{r}^{\prime}\right)$

where $E_{\text {ESW }}\left(\boldsymbol{r}, \boldsymbol{r}^{\prime}\right)$ is the complex valued ESW, $P(\boldsymbol{r})$ is the probe, and $O(r)$ is an object function. Here $r$ and $\boldsymbol{r}^{\prime}$ are 2D vectors in the $x, y$ plane, that define location of the scan position on the sample $\left(\boldsymbol{r}^{\prime}\right)$ and position within each single point of the scan $(\boldsymbol{r})$. The probe function is defined as an incident illumination on the colloidal crystal

$P(x, y)=E_{\text {in }}(x, y, z=0)$

The scattered amplitude $A\left(q_{x}, q_{\gamma}\right)$ in the far-field at each position of the sample $\left(\boldsymbol{r}^{\prime}\right)$ is determined by the Fourier transform of the ESW

$A_{\boldsymbol{r}^{\prime}}(\boldsymbol{q})=\int E_{\mathrm{ESW}}\left(\boldsymbol{r}, \boldsymbol{r}^{\prime}\right) \mathrm{e}^{-i \boldsymbol{q} \cdot \boldsymbol{r}} \mathrm{d} \boldsymbol{r}$ and the experimentally measured intensity is given by the square amplitude of this expression

$I_{r^{\prime}}(q)=\left|A_{r^{\prime}}(q)\right|^{2}$

In a thick colloidal film, the ESW may be determined by a multislice approach. ${ }^{[36,61,63]}$ In a thin colloidal film, the free-space propagation, which is represented by the propagation operator $\Delta_{\perp}$ in Equation (1), can be neglected, and the differential equation (1) gives relatively simple solution for the object function

$O(x, y)=\exp \left[i \frac{k}{2} \int_{0}^{\mathrm{d}(x, y)} \chi(x, y, z) \mathrm{d} z\right]$

The complex-valued susceptibility can be represented as a sum of real and imaginary parts

$\chi(x, y, z)=\chi^{\prime}(x, y, z)+i \chi^{\prime \prime}(x, y, z)$

and after substitution of Equation (7) in Equation (6), the complex valued object function can be written as

$O(x, y)=|O(x, y)| \mathrm{e}^{i \phi(x, y)}=\exp \left[-\frac{k}{2} \int_{0}^{\mathrm{d}(x, y)} \chi^{\prime \prime}(x, y, z) \mathrm{d} z\right] \exp \left[i \frac{k}{2} \int_{0}^{\mathrm{d}(x, y)} \chi^{\prime}(x, y, z) \mathrm{d} z\right]$

Here the amplitude of the object function is defined as

$|O(x, y)|=\exp \left[-\frac{k}{2} \int_{0}^{\mathrm{d}(x, y)} \chi^{\prime \prime}(x, y, z) \mathrm{d} z\right]$

The imaginary part of the susceptibility $\chi^{\prime \prime}(x, y, z)$ is related to the absorption coefficient $\mu(x, y)$ of the material by ${ }^{[64]}$

$\mu(x, y)=\frac{k}{2 d(x, y)} \int_{0}^{d(x, y)} \chi^{\prime \prime}(x, y, z) d z$

where $d(x, y)$ is the thickness of the crystal in $z$-direction. Thus, the amplitude of the object function (Equation (9)) can be written as

$|O(x, y)|=\mathrm{e}^{-\mu(x, y) d(x, y)}$

The phase $\phi(x, y)$ of the object function in the second term of Equation (8) is defined by

$\phi(x, y)=\frac{k}{2} \int_{0}^{d(x, y)} \chi^{\prime}(x, y, z) d z$

The real part of the susceptibility $\chi^{\prime}(x, y, z)$ is directly related to the electron density $\rho(x, y, z)$ by ${ }^{[64]}$

$\chi^{\prime}(x, y, z)=-\left[r_{e} \lambda^{2} / \pi\right] \rho(x, y, z)$

where $r_{\mathrm{e}}$ is the classical electron radius. Substituting Equation (13) in Equation (12), we obtain for the phase of the object function

$\phi(x, y)=-r_{\mathrm{e}} \lambda \int_{0}^{\mathrm{d}(x, y)} \rho(x, y, z) \mathrm{d} z=-r_{\mathrm{e}} \lambda R(x, y)$

where $R(x, y)=\int_{0}^{d(x, y)} \rho(x, y, z) d z$ is the projection of electron density $\rho(x$, $y, z)$ on the $x y$-plane taken along the beam propagation direction $z$. In ptychographic reconstruction, the amplitude equation (11) and phase 


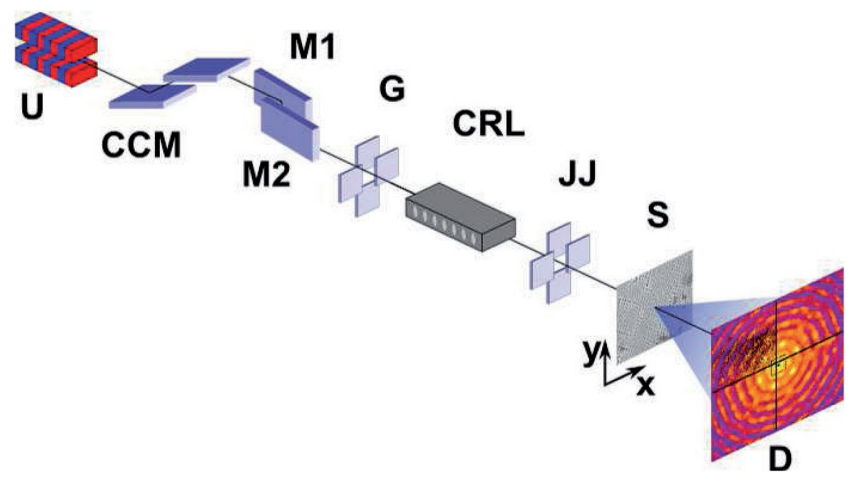

Figure 4. Overview of the experimental setup. The photon beam is generated by an undulator $(U)$ and traverses the beamline optics: channelcut monochromator (CCM), horizontal mirrors (M1, M2), a slit system $(G)$, compound refractive lenses (CRL), and a pair of slits $(J))$. The $X$-ray beam scatters on the sample $(S)$ and the resulting diffraction pattern is recorded in the far-field by the detector (D).

equation (12) are determined directly from the experimental data, for example, by fast converging alternating projections algorithm. ${ }^{[52]}$

Experimental Setup: The X-ray ptychography experiment on a colloidal crystal has been performed at the coherence beamline P10 of the PETRA III synchrotron at DESY, Hamburg with an incident X-ray photon energy of $8 \mathrm{keV}$. A schematic overview of the experimental setup is shown in Figure 4. Undulator radiation was monochromatized using a Si (111) channel-cut monochromator, and a pair of flat mirrors was used to reject higher harmonics. The monochromatic beam was focused on the sample (87.7 $\mathrm{m}$ from the source) using transfocator optics equipped with Be CRLs. ${ }^{[65]} \mathrm{A}$ set of three lenses with curvature radii of $50 \mu \mathrm{m}$ was used to focus the beam at the sample position $(1.57 \mathrm{~m}$ downstream the CRL) to a size of $2(v) \times 3(h) \mu \mathrm{m}^{2}$. The coherent illumination area at the entrance of the CRL optics was defined by a slit system (G) of $100(\mathrm{v}) \times 75(\mathrm{~h}) \mu \mathrm{m}^{2}$ in size located $2.1 \mathrm{~m}$ upstream the CRL. Additionally, a pair of slits $(J J)$ and a circular pinhole of $50 \mu \mathrm{m}$ in diameter were used as guard apertures in front of the sample. Diffraction patterns were collected using a MAXIPIX detector (ESRF, Grenoble, France) having an active area of $516 \times 516$ pixels $(2 \times 2$ arrangement with a single pad of $256 \times 256$ pixels) and a pixel size of $55 \mu \mathrm{m}$. The gap between adjacent chips of MAXIPIX results in a thin crosslike region of missing data in the measured diffraction patterns (Figure 2a). The detector was positioned $5.1 \mathrm{~m}$ downstream from the sample. The flight path between the sample and detector was evacuated in order to reduce background scattering on air. A $3 \times 3 \mathrm{~mm}^{2}$ semitransparent beamstop made of a $0.3 \mathrm{~mm}$ silicon wafer with calculated transmission ${ }^{[66]}$ of $1.3 \%$ was used to attenuate the intense small-angle scattering $X$-rays. The semitransparent beamstop effectively increased the dynamic range of the MAXIPIX detector. ${ }^{[67]}$ To protect the detector from the highly intense unscattered beam, an additional circular nontransparent Ta beamstop with a diameter of $0.5 \mathrm{~mm}$ was glued on top of the silicon square (see also ref. [50] for the details of experiment).

To determine the incident wave field on the colloidal crystal, ptychographic measurements using a Siemens star test gold pattern were performed first (see the Supporting Information for details). Next, the colloidal crystal sample was scanned over a $11 \times 11$ grid with $1 \mu \mathrm{m}$ step size in horizontal and vertical directions.

Samples: Colloidal crystals fabricated by the vertical deposition method were studied. ${ }^{[56,57,68]}$ PS spheres with a diameter of $418 \pm 9 \mathrm{~nm}$ were synthesized by polymerization of the aqueous solution of styrene using potassium persulfate as the initiator. Polydispersity of particles in colloidal suspension was $2.1 \%$ as measured by dynamic light scattering. Thin glass coverslips $(0.17 \mathrm{~mm}$ thickness) were inserted into colloidal suspensions contained particles at $1 \%$ volume fraction in water and subsequently dried at $323 \mathrm{~K}$ for several days. The grown colloidal crystal films consisted of 30-50 monolayers of PS spherical particles. Typically, dry colloidal crystal films exhibit a cracked texture consisting of single-crystal regions with an average size of 20-50 $\mu \mathrm{m}$. ${ }^{[53]}$ The same sample was also studied with high temperatures in melting conditions. ${ }^{[50]}$

\section{Supporting Information}

Supporting Information is available from the Wiley Online Library or from the corresponding author.

\section{Acknowledgements}

The authors would like to acknowledge E. Weckert for fruitful discussions and support of the project. The authors acknowledge a careful reading of the manuscript by D. Novikov. This work was partially supported by the Virtual Institute VH-VI-403 of the Helmholtz Association.

\section{Conflict of Interest}

The authors declare no conflict of interest.

\section{Keywords}

coherent X-ray diffraction imaging, colloidal crystals, domain structures, ptychography

Received: July 26, 2017

Revised: September 8, 2017

Published online: November 24, 2017

[1] J.-M. Meijer, A. Pal, S. Ouhajji, H. N. W. Lekkerkerker, A. P. Philipse, A. V. Petukhov, Nat. Commun. 2017, 8, 14352.

[2] É. Ducrot, M. He, G.-R. Yi, D. J. Pine, Nat. Mater. 2017, 16, 652.

[3] C. X. Du, G. van Anders, R. S. Newman, S. C. Glotzer, Proc. Natl. Acad. Sci. USA 2017, 114, E3892.

[4] M. C. Weidman, D.-M. Smilgies, W. A. Tisdale, Nat. Mater. 2016, 15, 775.

[5] J. J. Geuchies, C. van Overbeek, W. H. Evers, B. Goris, A. de Backer, A. P. Gantapara, F. T. Rabouw, J. Hilhorst, J. L. Peters, O. Konovalov, A. V. Petukhov, M. Dijkstra, L. D. A. Siebbeles, S. van Aert, S. Bals, D. Vanmaekelbergh, Nat. Mater. 2016, 15, 1248.

[6] A. Pal, V. Malik, L. He, B. H. Erne, Y. Yin, W. K. Kegel, A. V. Petukhov, Angew. Chem., Int. Ed. 2015, 54, 1803.

[7] A. V. Petukhov, J.-M. Meijer, G. J. Vroege, Curr. Opin. Colloid Interface Sci. 2015, 20, 272.

[8] W. Liu, N. A. Mahynski, O. Gang, A. Z. Panagiotopoulos, S. K. Kumar, ACS Nano 2017, 11, 4950.

[9] G. Van Anders, N. K. Ahmed, R. Smith, M. Engel, S. C. Glotzer, ACS Nano 2014, 8, 931.

[10] A. L. Thorneywork, J. L. Abbott, D. G. A. L. Aarts, R. P. A. Dullens, Phys. Rev. Lett. 2017, 118, 158001

[11] Q. Chen, S. C. Bae, S. Granick, Nature 2011, 469, 381.

[12] A. V. Petukhov, R. Tuinier, G. J. Vroege, Curr. Opin. Colloid Interface Sci. 2017, 30, 54.

[13] S. Arai, H. Tanaka, Nat. Phys. 2017, 13, 503.

[14] F. Wang, D. Zhou, Y. Han, Adv. Funct. Mater. 2016, 26, 8903.

[15] P. Tan, N. Xu, L. Xu, Nat. Phys. 2013, 10, 73. 
[16] A. F. Demirörs, P. J. Beltramo, H. R. Vutukuri, ACS Appl. Mater. Interfaces 2017, 9, 17238.

[17] Y. Yang, L. Gao, G. P. Lopez, B. B. Yellen, ACS Nano 2013, 7, 2705.

[18] I. Buttinoni, M. Steinacher, H. Th. Spanke, J. Pokki, S. Bahmann, B. Nelson, G. Foffi, L. Isa, Phys. Rev. E 2017, 95, 12610.

[19] A. Blanco, E. Chomski, S. Grabtchak, M. Ibisate, S. John, S. W. Leonard, C. Lopez, F. Meseguer, H. Miguez, J. P. Mondia, G. A. Ozin, O. Toader, H. M. van Driel, Nature 2000, 405, 437.

[20] Y. A. Vlasov, X.-Z. Bo, J. C. Sturm, D. J. Norris, Nature 2001, 414, 289.

[21] Q. Zhao, C. E. Finlayson, D. R. E. Snoswell, A. Haines, C. Schäfer, P. Spahn, G. P. Hellmann, A. V. Petukhov, L. Herrmann, P. Burdet, P. A. Midgley, S. Butler, M. Mackley, Q. Guo, J. J. Baumberg, Nat. Commun. 2016, 7, 11661.

[22] F. Fu, Z. Chen, Z. Zhao, H. Wang, L. Shang, Z. Gu, Y. Zhao, Proc. Natl. Acad. Sci. USA 2017, 114, 5900.

[23] A. Stein, B. E. Wilson, S. G. Rudisill, Chem. Soc. Rev. 2013, 42, 2763.

[24] S. Kawakami, A. Mori, K. Nagashima, M. Haraguchi, T. Okamoto, J. Cryst. Growth. 2017, 468, 740.

[25] J. Ge, Y. Hu, Y. Yin, Angew. Chem., Int. Ed. 2007, 46, 7428.

[26] X. Li, K. Lu, Nat. Mater. 2017, 16, 700.

[27] D. McDermott, C. J. O. Reichhardt, C. Reichhardt, Phys. Rev. E 2016, 93, 62607

[28] J. Hilhorst, A. V. Petukhov, Phys. Rev. Lett. 2011, 107, 95501.

[29] J. S. Yodh, Y. Wang, Y. Wang, X. Zheng, M. Weck, D. J. Pine, Nat. Commun. 2015, 6, 7253.

[30] W. L. Vos, M. Megens, C. M. van Kats, P. Boesecke, Langmuir 1997, 13,6004

[31] T. Li, A. J. Senesi, B. Lee, Chem. Rev. 2016, 116, 11128.

[32] J. Hilhorst, M. M. van Schooneveld, J. Wang, E. de Smit, T. Tyliszczak, J. Raabe, A. P. Hitchcock, M. Obst, F. M. F. de Groot, A. V. Petukhov, Langmuir 2012, 28, 3614.

[33] M. M. van Schooneveld, J. Hilhorst, A. V. Petukhov, T. Tyliszczak, J. Wang, B. M. Weckhuysen, F. M. F. de Groot, E. de Smit, Small 2011, 7, 804.

[34] A. Bosak, I. Snigireva, K. S. Napolskii, A. Snigirev, Adv. Mater. 2010, 22, 3256.

[35] D. V. Byelov, J.-M. Meijer, I. Snigireva, A. Snigirev, L. Rossi, E. van den Pol, A. Kuijk, A. Philipse, A. Imhof, A. van Blaaderen, G. J. Vroege, A. V. Petukhov, RSC Adv. 2013, 3, 15670.

[36] V. Kohn, I. Snigireva, A. Snigirev, J. Synchrotron Rad. 2014, 21, 1.

[37] J. Als-Nielsen, D. McMorrow, Elements of Modern X-Ray Physics, 2nd ed., Wiley, Singapore 2011.

[38] I. A. Vartanyants, O. M. Yefanov, X-ray Diffraction. Modern Experimental Techniques, (Eds.: O. H. Seeck, B. M. Murphy), Pan Stanford Publishing Pte. Ltd., Singapore 2015, pp. 341-384.

[39] J. Gulden, O. M. Yefanov, A. P. Mancuso, V. V. Abramova, J. Hilhorst, D. Byelov, I. Snigireva, A. Snigirev, A. V. Petukhov, I. A. Vartanyants, Phys. Rev. B 2010, 81, 224105.

[40] J. Gulden, O. Yefanov, A. Mancuso, R. Dronyak, A. Singer, V. Bernátová, A. Burkhardt, O. Polozhentsev, A. Soldatov, M. Sprung, I. A. Vartanyants, Opt. Express 2012, 20, 4039.

[41] A. G. Shabalin, J.-M. Meijer, R. Dronyak, O. M. Yefanov, A. Singer, R. P. Kurta, U. Lorenz, O. Y. Gorobtsov, D. Dzhigaev,
S. Kalbfleisch, J. Gulden, A. V. Zozulya, M. Sprung, A. V. Petukhov, I. A. Vartanyants, Phys. Rev. Lett. 2016, 117, 138002.

[42] J. M. Rodenburg, A. C. Hurst, A. G. Cullis, B. R. Dobson, F. Pfeiffer, O. Bunk, C. David, K. Jefimovs, I. Johnson, Phys. Rev. Lett. 2007, 98, 034801.

[43] J. M. Rodenburg, Adv. Imaging Electron Phys. 2008, 150, 87.

[44] P. Thibault, M. Dierolf, A. Menzel, O. Bunk, C. David, F. Pfeiffer, Science 2008, 321, 379.

[45] W. Hoppe, Acta Crystallogr. Sect. A 1969, 25, 495.

[46] W. Hoppe, Acta Crystallogr. Sect. A 1969, 25, 508.

[47] W. Hoppe, G. Strube, Acta Crystallogr. Sect. A. 1969, 25, 502.

[48] A. J. D'Alfonso, A. J. Morgan, A. W. C. Yan, P. Wang, H. Sawada, A. I. Kirkland, L. J. Allen, Phys. Rev. B 2014, 89, 064101.

[49] In a recent work ${ }^{[69]}$ ptychography was applied to reveal domain morphology of butterfly wings.

[50] E. A. Sulyanova, A. Shabalin, A. V. Zozulya, J.-M. Meijer, D. Dzhigaev, O. Gorobtsov, R. P. Kurta, S. Lazarev, U. Lorenz, A. Singer, O. Yefanov, I. Zaluzhnyy, I. Besedin, M. Sprung, A. V. Petukhov, I. A. Vartanyants, Langmuir 2015, 31, 5274.

[51] P. Thibault, A. Menzel, Nature 2013, 494, 68.

[52] S. Marchesini, Y.-C. Tu, H.-T. Wu, Appl. Comput. Harmon. Anal. 2016, 41, 815 .

[53] J.-M. Meijer, Colloidal Crystals of Spheres and Cubes in Real and Reciprocal Space, Springer, Switzerland 2015.

[54] J. H. Conway, N. J. A. Sloane, Sphere Packings, Lattices and Groups, Springer, New York 1999.

[55] P. G. Bolhuis, D. Frenkel, S.-C. Mau, D. A. Huse, Nature 1997, 388, 235.

[56] J. Hilhorst, V. V. Abramova, A. Sinitskii, N. A. Sapoletova, K. S. Napolskii, A. A. Eliseev, D. V. Byelov, N. A. Grigoryeva, A. V. Vasilieva, W. G. Bouwman, K. Kvashnina, A. Snigirev, S. V. Grigoriev, A. V. Petukhov, Langmuir 2009, 25, 10408.

[57] J.-M. Meijer, V. W. A. de Villeneuve, A. V. Petukhov, Langmuir 2007, 23, 3554.

[58] V. W. A. de Villeneuve, P. S. Miedema, J. M. Meijer, A. V. Petukhov, EPL 2007, 79, 56001.

[59] L. Meng, H. Wei, A. Nagel, B. J. Wiley, L. E. Scriven, D. J. Norris, Nano Lett. 2006, 6, 2249.

[60] J. Gulden, O. M. Yefanov, E. Weckert, I. A. Vartanyants, AIP Conf. Proc. 2011, 1365, 42

[61] V. G. Kohn, N. V. Tsvigun, Crystal. Rep. 2014, 59, 1.

[62] P. Thibault, M. Dierolf, O. Bunk, A. Menzel, F. Pfeiffer, Ultramicroscopy 2009, 109, 338.

[63] J. M. Cowley, Diffraction Physics North-Holland Publishing Co., Amsterdam 1975

[64] A. Authier, Dynamical Theory of X-Ray Diffraction, Oxford University Press, Great Britain 2003.

[65] A. V. Zozulya, S. Bondarenko, A. Schavkan, F. Westermeier, G. Grübel, M. Sprung, Opt. Express 2012, 20, 18967.

[66] B. L. Henke, E. M. Gullikson, J. C. Davis, At. Data Nucl. Data Tables 1993, 54, 181.

[67] R. N. Wilke, M. Vassholz, T. Salditt, Acta Crystallogr. Sect. A 2013 , 69, 490.

[68] P. Jiang, J. Bertone, K. Hwang, V. Colvin, Chem. Mater. 1999, 11, 2132.

[69] A. Singer, L. Boucheron, S. H. Dietze, K. E. Jensen, D. Vine, I. McNulty, E. R. Dufresne, R. O. Prum, S. G. J. Mochrie, O. G. Shpyrko, Sci. Adv. 2016, 2, e1600149. 